\title{
Ein Nachruf für einen Freund
}

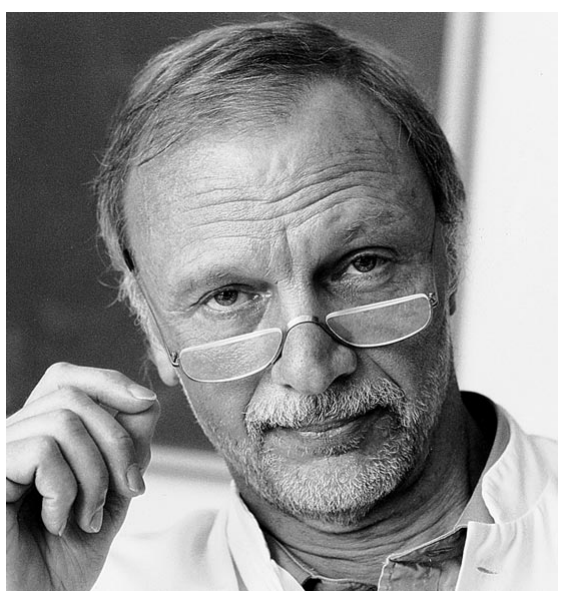

Prof. Dr. med. Karl-Ludwig Neuhaus geboren 17. 07. 1944, gestorben 15. 03. 2000 Direktor der Med. Klinik II im Städt. Klinikum Kassel

Vorsitzender der ALKK 1990-1994

Studienkoordinator der ALKK 1988-2000

Präsident-elect der DGK 2001-2003
Wo Sprachlosigkeit ihren Platz hat, werden Worte zum Risiko.

Dies ist eine Situation, in welcher Neuhaus sagte:

„Es kann immer etwas schiefgehen, wenn man etwas tut, das Schlimmere ist, etwas nicht zu tun".

So will ich versuchen, Gedanken auszusprechen, die viele mit Lutz Neuhaus auf einem gemeinsamen Stück Weg verbunden haben.

Geboren wurde er als Sohn eines Landarztes im Sauerland nach zwei Schwestern. Wer ihn kannte, kann sich nicht vorstellen, dass er pflegeleicht gewesen sein könnte. Jedenfalls muss er ein Elternhaus gehabt haben, das funktioniert hat. Nur so konnte er das Urvertrauen entwickeln und das traumwandlerische, sichere Urteilsvermögen, das viele an ihm bemerkten.

Lutz Neuhaus wollte es wissen.

Was sich in seiner Jugend wohl eher stürmisch offenbarte, war beim reiferen Neuhaus sublimiert in genuines Interesse an der Sache. Neuhaus machte es sich nie leicht, nicht im Privaten und nicht im Beruf. Sein Leben war reich an dem, was mein gemeinhin „Schicksal“ nennt und reich an Glück.

Was bleibt von dem Glück, sind seine Frau und drei Töchter, die er alle innig liebte. Derjenige, den er gelegentlich in sich hineinschauen ließ, war beeindruckt von seiner menschlichen Zuwendung und seiner hohen Achtung für andere.
In persönlichen Dingen war Lutz Neuhaus äußerst bescheiden. Er machte sich verdächtig als Asket: Ein Nahrungsmittel-Exzess für ihn war ein halber Laib Brot und ein Liter Milch. Er hatte Freude an der schnellen Bewegung auf Rädern gelegentlich sah man die Folgen.

Aus größerer Entfernung galt er als unbequem. Ihm war kein Gegner zu groß. Wer sich mit Neuhaus einließ, musste umdenken. Er war kompromisslos in seiner Gradlinigkeit, galt als Sozialethiker und als fanatischer Verfechter einer Wahrheit und einer Gerechtigkeit als Prinzip.

Für die jeweils andere Seite galt er als schwierig und - Zitat: „Der Mensch mit dem kranken Verhältnis zum Geld“. Das Schlimme für die andere Seite war häufig: Neuhaus behielt Recht. Obwohl ausgestattet mit sehr hoher menschlicher, intellektueller und fachlicher Kompetenz, blieb ihm ein Ordinariat verwehrt. Es schien Übereinstimmung darüber zu herrschen, dass er zu viel Unruhe in ein System bringen würde, das doch geordnet schien. Dies behinderte Neuhaus aber keinesfalls, internationale fachliche und wissenschaftliche Geltung zu erlangen.

Er fungierte als wissenschaftlicher Berater am National Institute of Health. Er war hochgeachtet in den Gremien der europäischen und amerikanischen kardiologischen Fachgesellschaften. Der Gold-Standard für die Lyse-Behandlung des akuten In- 
farktes trägt in der englischen Literatur den Namen „Neuhaus-Schema" und überlebt den Namensgeber nun in Braunwalds Lehrbuch.

Die Akronyme seiner multizentrischen Studien zur Infarktbehandlung GAUS, TAPS, GRECO und HIT lesen sich wie psychographische Daten eines wissenschaftlichen Lebens. Seine letzte große, wissenschaftliche Aufgabe war ein Modellprojekt zur Qualitätssicherung bei der Koronardilatation, mit Anschub-Finanzierung durch das Bundesministerium für Forschung und Technik und das Bundesministerium für Gesundheit.

Wie so vieles, das Neuhaus in die Hand nahm, war dieses Projekt

\section{Karl-Ludwig Neuhaus}

Nach dem Abitur 1963, in Wipperfürth, verbrachte Lutz Neuhaus seine vorklinischen Semester in Münster und Würzburg und wechselte dann für die Klinischen Semester an die Universität Düsseldorf, wo er 1969 auch das Staatsexamen ablegte.

Die Begeisterung für die Kardiologie entwickelte sich während der klinischen Semester in Düsseldorf unter Prof. Dr. Heinrich Kreuzer, von dem er auch das DissertationsThema „Die kontinuierliche Messung des koronarvenösen Sauerstoffdrucks mit einer nicht-membranbezogenen Platin-Elektrode" erhielt.

Nach der Medizinal-Assistentenzeit in Emmerich, am Niederrhein, und der Approbation Ende 1970 wechselte Neuhaus 1971 für ein Jahr als wissenschaftlicher Mitarbeiter an das Centre d'Etudes et deTechniques Chirurgicales nach Paris und begegnete dort Herrn Prof. Dr. S. Hagl, dem derzeitigen Präsidenten der Deutschen Gesellschaft für Thorax-Herz-Gefäßchirurgie, mit dem ihn eine lebenslange Freundschaft verband.

Von 1972 bis 1975 war Lutz Neuhaus wissenschaftlicher Assis- so überzeugend in der Logik seiner Konzeption, seiner Einfachheit und so durchschlagend im Erfolg, dass es nun auch von der Welt-Gesundheits-Organisation entdeckt wurde.

Das Gewicht von Neuhaus mit „Arbeit in Deutschland“ wog so schwer, dass eine Wahl zum Präsidenten der Deutschen Gesellschaft für Kardiologie unabwendbar schien.

Er wurde gewählt und hätte das Amt im Jahre 2001 antreten sollen.

Während Neuhaus starb, wurde eine seiner Studien zur PTCA als Infarkt-Therapie beim American College of Cardiology als „Hotline“ vorgetragen. Diese Studie wurde von vielen von uns als dringend notwendig, aber undurchführbar angesehen. Auch hier gab er eine Antwort zu einer Frage, die sich jeder Kardiologe einmal stellen musste, die aber kein anderer beantwortete, weil sie unbequem war.

Lutz Neuhaus kam zur Ruhe vor uns allen, obwohl er eigentlich noch gar nicht müde war.

Er ist gegangen am 15. März mit noch nicht 56 Jahren. Er ging von zu Hause, aus seiner Familie. Er hinterlässt das Bild eines würdigen Menschen.

Martin Gottwik, Nürnberg im März 2000 tent an der Medizinischen Klinik der Universität Düsseldorf, unter der Leitung von Prof. Dr. Franz Loogen. Nach dem Weggang von H. Kreuzer leitete Neuhaus bereits in jungen Jahren, zusammen mit Paul Spiller, von 1975 bis Ende 1977 das Projekt „Quantitative Röntgenologie des Herzens “ im Sonderforschungsbereich 30 der Deutschen Forschungsgemeinschaft, in Düsseldorf.

\section{Universitätsklinik Göttingen 1978-1987}

Karl-Ludwig Neuhaus wechselte aus der Düsseldorfer Kardiologie an die Kardiologische Klinik der GeorgAugust-Universität Göttingen, unter Leitung von Prof. Dr. Heinrich Kreuzer, und nahm zum 1. Januar 1978 eine Oberarzt-Stelle im wenige Monate vorher in Betrieb genommenen, neuen Universitäts-Klinikum an und habilitierte sich 1979 mit dem Thema „Ventrikel-Geometrie und myokardiale Arbeitsbedingungen bei hypertrophierten und dilatierten linken Ventrikeln“.
In Göttingen traf Neuhaus auf die Arbeitsgruppe um K.P. Rentrop, die sich mit der invasiven Akut-Diagnostik und -Therapie des akuten Myokardinfarktes beschäftigte.

In der Folgezeit entwickelte sich ein akademischer Wettstreit um neue Therapiekonzepte in der akuten Infarktbehandlung, der Anfang der 80er Jahre in der Frage gipfelte, ob die intrakoronare oder intravenöse Thrombolyse-Behandlung mit Streptokinase der bessere Weg zur Rekanalisation thrombotisch verschlossener Koronargefäße ist. In Zusammenarbeit mit dem Hämostaseologen Heinz Köstering hatte Neuhaus Anfang der 80er Jahre die ,hochdosierte Streptokinase-Kurzzeit-Lyse“ im Rahmen einer Dosisfindungs-Studie untersucht und war zu der Erkenntnis gekommen, dass eine Dosierung von ca. 2 Mio. IE Streptokinase bei intravenöser Applikation die besten Rekanalisations-Raten thrombotisch verschlossener Koronararterien ergab (Clin. Cardiol. 1983, 6:426-434).

Etwa gleichzeitig führte Rolf Schröder in Berlin ähnliche intravenöse Streptokinase-Lysen beim aku- 
ten Myokardinfarkt durch, die bei Dosierungen zwischen 0,5 und 1,0 Mio. IE auch gute Patency-Raten zeigten. Im Rahmen eines „LyseGipfels“ 1981, in Göttingen, trafen sich Neuhaus und Schröder etwa auf der Mitte und legten die intravenöse Gabe von 1,5 Mio. IE Streptokinase in einer Stunde als optimale Dosierung fest, was später als „Gold-Standard“ der StreptokinaseLyse beim akuten Myokardinfarkt in die Literatur eingehen sollte. Die weitere Zusammenarbeit von Neuhaus und Schröder mündete in der zwischen 1982 und 1985 durchgeführten ersten größeren, randomisierten, doppelblinden und placebokontrollierten Streptokinase-LyseStudie beim akuten Myokardinfarkt, die unter dem Akronym ISAM-Studie ebenfalls in die kardiologische Weltliteratur eingegangen ist (NEJM 1986, 314:1465-1471).

Nach diesen Erfahrungen mit einer nach modernen Gesichtspunkten (doppelblinde, placebokontrollierte Randomisation, unabhängige Statistik, Sponsering durch Industrie und Bundesministerien, Kontroll-Komitee und Ethik-Voten usw.) durchgeführten Therapie-Studie wandte sich Neuhaus dann folgerichtig und konsequent der neuen, erstmalig gentechnologisch entwickelten thrombolytischen Substanz des Gewebs-Plasminogen-Aktivators zu und führte zunächst eine Dosis-Findungs-Studie durch, wobei - typisch für Lutz Neuhaus - als VergleichsSubstanz die bisher noch nicht so gut untersuchte, aber vermeintlich sogar bessere Substanz Urokinase ausgesucht wurde.

Auch das Ergebnis dieser wiederum multizentrischen Dosis-Findungs-Studie fand unter der Abkürzung GAUS internationale Beachtung (JACC 1988, 12:581-587).

Parallel mit seinem Wechsel an die Städtischen Kliniken Kassel wurde in Kooperation mit seiner alten Wirkungsstätte, in Göttingen, eine optimierte Dosierung des Gewebsplasminogen-Aktivators (rtPA) an Infarktpatienten mit nachgewiesenem, thrombotischen Koronarverschluss untersucht (JACC 1989, 14:1566-1569). Diese von der anglo-amerikanischen Literatur als „Front-loaded rtPA“ oder „NeuhausRegime" bezeichnete Dosierung von Alteplase wurde in der Folgezeit in vielen Mega-Trials (GUSTO I und III, ASSENT-2, InTIME-2) getestet, aber bisher nicht übertroffen.

Neben dieser wissenschaftlichen Tätigkeit im Rahmen der Infarktforschung setzte sich Neuhaus mit noch größerer Intensität für die kli- nische Versorgung der Patienten im Göttinger Universitäts-Klinikum ein. Neben seiner begnadeten manuellen Fähigkeit in der invasiven kardialen Diagnostik und interventionellen Therapie war sein scharfer analytischer Intellekt bei allen Fachabteilungen des Göttinger Klinikums gefragt. Als ständiger Konsiliarius war Neuhaus zu jeder Tages- und NachtZeit zu Interventionen, insbesondere bei intensivmedizinischen Fragen der anderen Fachdisziplinen bereit.

Dieses Engagement führte zu allgemein höchster Akzeptanz und Bewunderung. Dies strahlte insbesondere auch auf die in der Ausbildung befindlichen AssistenzärzteInnen der Kardiologischen Abteilung aus und führte zu einer vielfach freundschaftlichen Verbundenheit, weit über die Göttinger Zeit hinaus.

Der Tod von Lutz Neuhaus hinterlässt bei vielen Weggefährten eine nicht zu schließende Lücke, seine menschliche Art, seine Kreativität, seine analytische Intelligenz und sein Engagement für die Klinische Kardiologie wird für die ,Göttinger Kardiologen und Pulmologen“ immer Vorbild bleiben.

Ulrich Tebbe, Detmold im März 2000

\section{Pressemitteilung des Klinikums Kassel}

Herr Professor Dr. med. Karl-Ludwig Neuhaus, Direktor der Medizinischen Klinik II im Klinikum Kassel ist nach kurzer schwerer Krankheit am 15. März 2000 verstorben.

Das Klinikum Kassel trauert um einen hervorragenden Mediziner. Sowohl von seinen Kollegen als auch von seinen Mitarbeitern wurde er sehr geschätzt. Herr Professor Neuhaus hat die von ihm geführte Klinik in den 13 Jahren seiner Tätigkeit zu einem bedeutenden und anerkannten kardiologischen Zen- trum mit internationalem Ruf entwickelt.

Im Frühjahr 1987 hatte Professor Neuhaus die Medizinische Klinik als Nachfolger des aus Altersgründen ausgeschiedenen Professor Dr. med. R. Heinecker übernommen. Als Direktor der Medizinischen Klinik II konnte Professor Neuhaus aufgrund der schon vorhandenen medizinischen Infrastruktur sowie der personellen und apparativen Ausstattung zu einem sehr frühen Zeitpunkt, nicht zuletzt durch sehr großes persönliches Engagement, seine Klinik so organisieren, dass eine kompetente und umfassende $\mathrm{Di}$ agnostik und Therapie akuter lebensbedrohlicher Herzerkrankungen in einer 24-Stunden-Bereitschaft möglich war.

Im Klinikum Kassel führte er mit großer Umsicht und zukunftsorientiert die ersten Koronararteriendilatationen und die Wiedereröffnung verschlossener Herzkranzgefäße als Notfallmaßnahme beim akuten Herzinfarkt sowie die Sprengung 
verengter Mitral- und Aortenklappen durch, was seinerzeit in Fachkreisen, wegen des vermeintlich hohen Risikos ohne eine herzchirurgische Hintergrundbereitschaft in unmittelbarer Nähe, als sehr kritisch beurteilt wurde. Nicht zuletzt wegen der hier gewonnenen positiven Erfahrungen und der Darstellung der guten Ergebnisse, verbunden mit der Beteiligung an der rasanten technischen Weiterentwicklung der Kathetertechnologien sind diese Maßnahmen heute in entsprechenden Zentren zur täglichen Routine geworden.

Den vermeintlichen Nutzen neuer Methoden und Maßnahmen überprüfte er stets mit interner selbstkritischer Qualitätskontrolle.

Unermüdlich setzte sich Professor Neuhaus für die Einrichtung einer Klinik für Herz-, Thorax- und Gefäßchirurgie im Klinikum ein, um die Versorgung herzerkrankter Patienten von der Diagnostik bis zur endgültigen operativen Behandlung auf kürzestem Wege und ohne Zeitverlust sicherstellen zu können. 1993 erfüllte sich sein Wunsch in Form der Einrichtung einer gut ausgestatteten kardiochirurgischen Klinik. Getragen von seiner Fachkompetenz und Kooperationsfähigkeit gelang die rasche und reibungslose konzeptionelle Umsetzung eines funktionstüchtigen kardiologischkardiochirurgischen Zentrums vor Ort, dessen Ruf weit über die Grenzen Hessens hinausgeht.

Neben der Kardiologie galt sein besonderes Interesse der internistischen Intensivmedizin. Die Inbetriebnahme der unter seiner Leitung über einen langen Zeitraum geplanten Erweiterung und den Umbau der Medizinischen Intensivstation mit modernsten Überwachungs- und Behandlungsmöglichkeiten sowie die Eröffnung der, ihm so wichtigen, medizinischen Notaufnahmestation durfte er noch in vollem Umfang miterleben.

In der von ihm ausgebauten Institutsambulanz suchten viele Patienten mit komplexen kardiologischen Problemen seinen Rat und seine Hilfe.
Schon zu einem sehr frühen Zeitpunkt führte er auf hohem Niveau ambulante Katheteruntersuchungen des linken und rechten Herzens durch. Diese, bis dahin in der nordhessischen Region außergewöhnliche Einrichtung wurde von regionalen und überregionalen Krankenhäusern sowie niedergelassenen Kollegen hoch geschätzt und regelmäßig genutzt. Dies belegen die vielen Patienten, die in die Klinik überwiesen werden.

Neben den ungezählten Vorträgen zur kardiologischen Fortbildung niedergelassener Kollegen und Klinikärzte in ganz Deutschland organisierte und leitete er regelmäßig über annähernd 11 Jahre die „Kasseler Kardiologischen Gespräche“, eine angesehene Veranstaltung mit Seminarcharakter, die aufgrund der offenen Diskussionsmöglichkeit intensiven regionalen und überregionalen ärztlichen Zuspruch fand und eine sehr positive Darstellung seiner Klinik nach außen zur Folge hatte.

Unter seinem, von Selbstdisziplin und mitmenschlicher Verbindlichkeit geprägten, Führungsstil erwarben sich eine große Zahl ärztlicher Mitarbeiter ihre fundierte internistische Facharztweiterbildung sowie das kardiologisch-technische Rüstzeug zur selbstständigen und eigenverantwortlichen Tätigkeit.

Zudem begleitete er eine Reihe junger Ärzte erfolgreich durch das Promotionsverfahren und motivierte seine engsten Mitarbeiter zu selbständiger wissenschaftlicher Arbeit, was für einen außeruniversitären Klinikleiter in dieser Intensität auBerordentlich war.

Neben der Klinikleitung gab er sich unermüdlich der wissenschaftlichen medizinischen Forschung hin.

Vor dem Hintergrund, dass die hochentwickelte Medizintechnik nur an speziellen Zentren für wenige $\mathrm{Pa}$ tienten verfügbar sein würde, widmete er sein Wissen und seine Schaffenskraft über die ganzen Jahre hin einer flächendeckenden modernen Herzinfarkttherapie.

Unter seiner Anregung, Beteiligung und Leitung wurden seit
1987 von Kassel aus eine Reihe von Studien mit bekannten und neu entwickelten thrombolytisch wirkenden Medikamenten durchgeführt.

Die große Zahl der veröffentlichten Forschungsergebnisse in hochrangigen Fachzeitschriften, in Buchbeiträgen und in Referaten anlässlich der jährlichen Kongresse der Deutschen und Europäischen Gesellschaft für Kardiologie sowie der beiden großen amerikanischen kardiologischen Fachgesellschaften (AHA und ACC) fanden nationale und internationale Anerkennung und machten seine Person und das Klinikum Kassel weltweit bekannt.

Professor Neuhaus war es bis zuletzt ein besonderes Anliegen, die gesundheitspolitisch gewollten und gesetzlich vorgeschriebenen Qualitätskontrollen diagnostischer und therapeutischer Maßnahmen konsequent und praktikabel im klinischen Alltag durchzuführen. Dies wurde von ihm nicht als lästige $\mathrm{Zu}$ satzaufgabe gesehen, sondern als Möglichkeit zur eigenen Erfolgskontrolle und damit auch zu einer rationellen Optimierung der Patientenversorgung begriffen.

Diese Vorstellungen konnte er als Mitbegründer der 1988 gebildeten „Arbeitsgemeinschaft leitender kardiologischer Krankenhausärzte“ (ALKK), einem Zusammenschluss von derzeit 143 leitenden Krankenhausärzten in ganz Deutschland, die in ihren Institutionen über das gesamte Spektrum der invasiven, nichtinvasiven, intensivmedizinischen, stationären und ambulanten Behandlungsmethoden verfügen einbringen. Er war als Vorsitzender und Sekretär dieser Arbeitsgemeinschaft maßgeblich an der Formulierung der Zielsetzungen und Aufgaben, nämlich der Durchführung praxisrelevanter kardiologischer Versorgungsforschung beteiligt.

Unter seiner Leitung wurden schon einige Teilprojekte in Form von Patientenregistern über das Klinikum Kassel als Referenzzentrum für ganz Deutschland organisiert, betreut und ausgewertet. Die hier in kürzester Zeit 
gewonnenen und bisher einmaligen Studienergebnisse haben teilweise schon unmittelbaren Einfluss auf zukünftige Richtlinien zur optimierten und gleichzeitig ökonomisch vertretbaren Patientenversorgung.

Als ausgewiesener und kompetenter Spezialist auf diesem Gebiet galt er als kritischer und unparteiischer Berater verschiedenster sozial- und gesundheitspolitischer Gremien.

Im Klinikum Kassel war Herr Professor Neuhaus stellvertretender Ärztlicher Direktor und sollte im Jahre 2001 die Stelle des Ärztlichen Direktors übernehmen. Er war schon in seiner derzeitigen Funktion in alle wesentlichen Strukturentwicklungen aktiv eingebunden und hatte zurückliegende und anstehende Entscheidungen in hohem Maße positiv beeinflusst. Er zeichnete sich im Direktorium durch seine Sachkenntnis und seine fächerübergreifende $\mathrm{zu}-$ kunftsorientierte Einstellung aus, was im Kollegenkreis außerordentlich anerkannt und geschätzt wurde.

In Anerkennung seiner bisherigen Verdienste war Herr Professor Neuhaus gewählter und designierter Anwärter auf die Präsidentschaft der Deutschen Gesellschaft für Kardiologie (DGK).
Er sollte das von ihm sehr geschätzte, ehrenvolle Amt zu Anfang des Jahres 2001 antreten.

Mit ihm hat nicht nur das Klinikum Kassel eine außergewöhnliche Persönlichkeit sowie Mediziner verloren.

Sein medizinischer Weitblick, seine kritischen Stellungnahmen sowie seine hochqualifizierten wissenschaftlichen Beiträge zur Realisierung einer „effizienten“ und ,praktikablen“ Medizin werden uns fehlen.

Wolfgang Schäfer, Geschäftsführer, Klinikum Kassel 